\title{
Research Paper \\ The Effects of an Eight-week Cawthorne-Cooksey Training Program on Bal- ance and Lower Limb Strength in the Elderly
}

\author{
Mohammad Javad Azimizadeh ${ }^{1}$ (1), *Seyyed Mojtaba Tabatabai AsI ${ }^{1}\left(\mathbb{C}\right.$, Seyyed Hosein Hoseini $^{1}{ }^{(1)}$
}

1. Department of Sport Injuries and Corrective Exercise, Faculty of Physical Education and Sport Sciences, University of Guilan, Rasht, Iran.

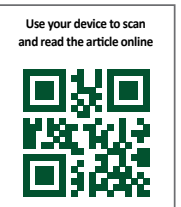

Crtation: Azimizadeh MJ, Tabatabai Asl SM, Hoseini SH. [The Effects of an Eight-week Cawthorne-Cooksey Training Program on Balance and Lower Limb Strength in the Elderly (Persian)]. Journal of Sport Biomechanics. 2021; 7(1):68-77. https:// doi.org/10.32598/biomechanics.7.1.6

doi'https://doi.org/10.32598/biomechanics.7.1.6

Keywords:

Balance, Lower Limbs,

Aged, Cawthorne-

Cooksey

\section{ABSTRACT}

Objective With age, the problems associated with loss of balance, followed by decreased muscle strength and physical function, increase. Therefore, the elderly are exposed to serious injuries. Therefore, the aim of the present study was to investigate the effect of Cawthorne Cooksey training on balance and lower limb strength in the elderly.

Methods Subjects including 24 elderly men and women participated in this study voluntarily and were divided into experimental groups $(n=12)$ and control $(n=12)$. The design of the present study was pre-test and post-test. Cawthorne Cooksey training were performed for eight weeks, three sessions per week and each session for 60 minutes. The main variables were balance and lower limb strength, and the leaf test was used to assess balance and the rise and fall test was used for lower limb strength. One-way covariance and dependent t-test in SPSS software version 26 were used to analyze the pre-test and post-test results and compare the groups.

Results The results of this study showed that the balance in the experimental group had a significant improvement compared to the control group $(\mathrm{P}=0.001)$. There was also a significant difference in lower limb strength in the experimental group compared to the control $(\mathrm{P}=0.002)$.

Conclusion According to the findings of this study, these exercises positively affect balance and lower limb strength in the elderly. Due to the decline in physical function of the elderly, performing these exercises increases the level of physical function and quality of life of the elderly.

\section{Extended Abstract}

\section{Introduction}

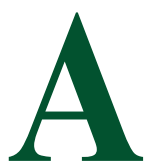

ging is a natural and fast process, i.e., achieved as a result of leaving behind childhood, adolescence, youth, as well as middle age. Aging is an undeniable aspect of life. Decreased birth rates, improved health status, and enhanced life expectancy have led to an increase in life expectancy and consequently an increase in the elderly or the phenomenon of aging, globally [1]. With the onset of old age, changes occur in balance-related physiological systems. The balance control system is a complex mechanism in which coordination between balance systems plays an important role [2]. Common problems in the elderly that occur as a result of aging or illness are loss of balance and postural control. With aging, physical changes or fluctuations increase; consequently, the odds of falling elevated in this population [3].

\section{* Corresponding Author:}

Seyyed Mojtaba Tabatabai Asl

Address: Department of Sport Injuries and Corrective Exercise, Faculty of Physical Education and Sport Sciences, University of Guilan, Rasht, Iran.

Tel: +98 (915) 6278418

E-mail: syd.mojtaba75@gmail.com 
Table 1. The anthropometric data of the study groups

\begin{tabular}{|c|c|c|}
\hline \multirow{2}{*}{ Characteristic } & \multicolumn{2}{|c|}{ Mean $\pm S D$} \\
\hline & Test Group (n=12) & Control Group ( $n=12$ ) \\
\hline Age, $y$ & $65.53 \pm 2.95$ & $67.12 \pm 1.62$ \\
\hline Height, cm & $169.90 \pm 6.47$ & $168.04 \pm 5.17$ \\
\hline Weight, kg & $68.10 \pm 9.87$ & $67.31 \pm 7.24$ \\
\hline
\end{tabular}

\section{Methods}

This was a quasi-experimental and applied study. The required study sample was selected from the elderly volunteers referring to the elderly centers of Mashhad City, Iran by the convenience sampling method. In total, 24 elderly men and women were divided into two groups of 12 individuals. Explanations on how to perform the exercises were provided by the researcher to the study subjects. Furthermore, after providing the informed consent form, the research subjects started the exercises. The Berg Balance Scale (BBS) was used to assess balance (internal validity: 0.98, external validity: 0.99), and the Timed Up and Go (TUG) test was used to evaluate lower limb strength (reliability: 0.99) in the study participants.

After performing the pretest measurements, the experimental group performed Cawthorne-Cooksey Training Program (CCTP) 3 times a week for 2 months. After 8 weeks of training, the study subjects were re-evaluated by the BBS and TUG test. The normality of the data was evaluated using the Kolmogorov-Smirnov test. Dependent Samples t-test was used to compare the differences between pretest and posttest stages. Moreover, Analysis of Covariance (ANCOVA) was used to compare the differences between the research groups. The obtained data were analyzed in SPSS at the significance level of $\mathrm{P}<0.05$.

\section{Results}

The results of Shapiro-Wilk test confirmed the normality of the data concerning the variables of balance index and lower limb strength. The data respecting the demographic information and the effects of CCTP on balance and lower limb strength in the elderly are presented in Table 1 . The anthropometric data of the study groups is provided in Table 1.

As per Table 1, in the pretest stage, there was no significant difference in the balance and lower limb strength index; thus, the study groups were homogeneous in this area. The ANCOVA data on the difference between the scores of these indicators in the posttest revealed a significant difference between the control and experimental groups in the balance and strength index of the lower limbs. Furthermore, the Dependent Samples t-test results indicated that the experimental group had a significant improvement in the balance and strength index of the lower limbs, compared to the pretest values $(\mathrm{P}<0.05)$.

\section{Discussion and Conclusion}

The present study investigated the effects of CCTP on balance and lower limb strength in the elderly. The obtained results indicated that CCTP was effective in improving balance and lower limb strength of the elderly in the experimental group, compared to the control group $(\mathrm{P}<0.05)$. Moreover, there was a significant difference in the pretest and posttest stages between the experimental groups of static and dynamic balance. Song et al. (2016) investigated the effects of trunk and neck stability exercises on balance among the elderly; accordingly, the related results reflected a significant increase in balance records [15].

Bratchman et al. (2015) conducted a study on the therapeutic effect of CCTP on balance development and fear of falling in the elderly. Another study positively assessed the effects of CCTP on improving balance and postural control, functional capacity, and quality of life in the elderly [16]. Abarghaei et al. (2018) investigated the effects of CCTP on balance and quality of life in the elderly with an age range of 60-80 years. They concluded that CCTP significantly affected balance and quality of life in the elderly due to complete involvement of the atrial and ocular systems [17], i.e., consistent with the present study findings.

Additionally, the findings of this study were in line with those of Farnada et al. (2016), Kamrani Faraz et al. (2016), and Mirzaeian et al. (2016) who reported the improvement of lower limb strength after exercise programs [18-20]. Zambar et al. (2015) explored the influence of CCTP on static and dynamic balance and the odds of falls in older women. In their study, they examined subjects were divid- 
ed into two groups of routine treatment and CCTP. After the intervention, they found that in the CCTP group, static and dynamic balance, as well as the odds of falling, were significantly improved [21]. The dynamism of some exercise items also facilitates improving the lower limb strength of the research subjects. Furthermore, the BBS alone cannot express balance and strength; thus, the TUG test was used.

\section{Ethical Considerations}

Compliance with ethical guidelines

All ethical principles are considered in this article. The participants were informed about the purpose of the research and its implementation stages. They were also assured about the confidentiality of their information and were free to leave the study whenever they wished, and if desired, the research results would be available to them.

Funding

This research did not receive any grant from funding agencies in the public, commercial, or non-profit sectors.

Authors' contributions

All authors equally contributed to preparing this article.

Conflicts of interest

The authors declared no conflict of interest.

Acknowledgements

The author would like to thank all staff of Mashhad Health Nursing Home for their help. 
مقاله يُورهشى

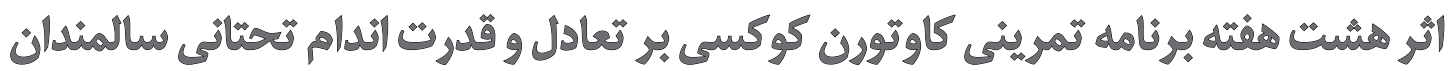

\author{
محمد جواد عظيمىزاده' هـ، "سيد مجتبى طباطبايى اصل' ه، سيد حسين حسينى' (1) \\ 1. كروه آسيب شناسى ورزشى وحركات اصلاحى، دانشكده تربيت بدنى و علوم ورزشى، دايشكاه كيلان، رشت، ايران.
}

\begin{abstract}
حكند

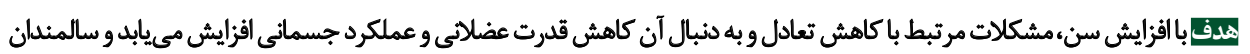

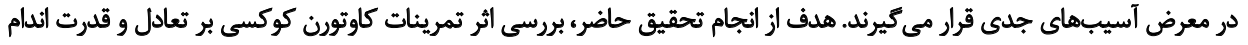
تحتانى سالمندان بود.

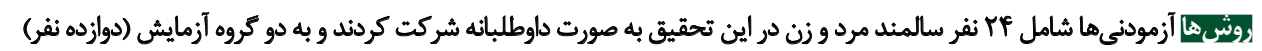

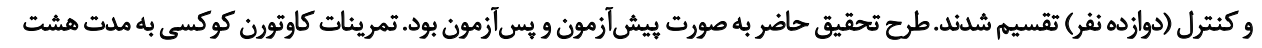

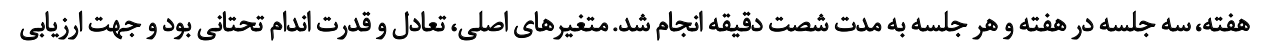

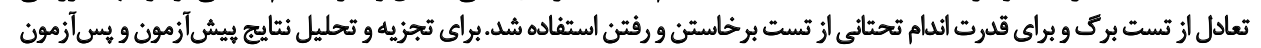

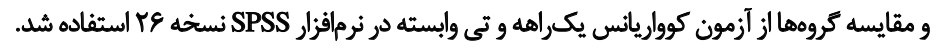

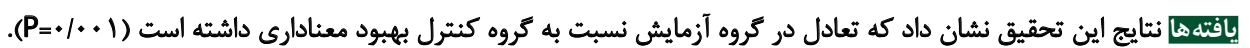

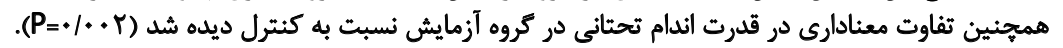

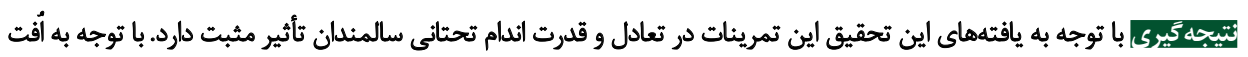

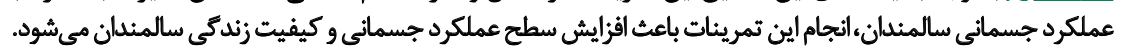

اطلاعات مقاله: - إن

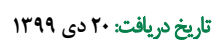

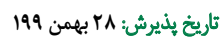

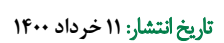

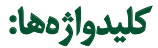
تعادل، اندام تصنتأئي، سالمندان، تمرينات كوكسى كاوتون تمرنين

احتمال زمين خوردن اين افراد افزايش مىيابد [ب].

doles

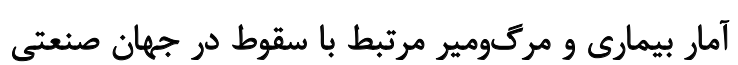

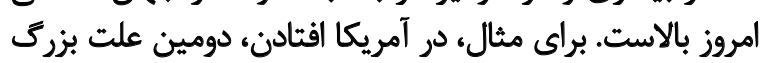

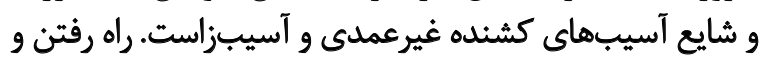

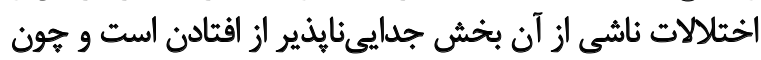

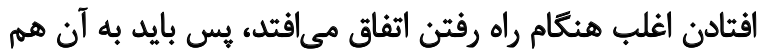
توجه خاصى داشت.

برخى محققين معتقدند كه كاهش تعادل به عنوان اصلى ترين

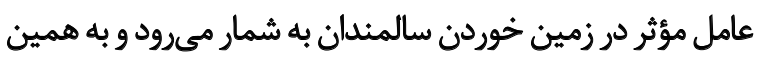

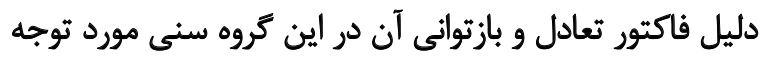

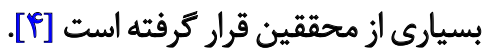

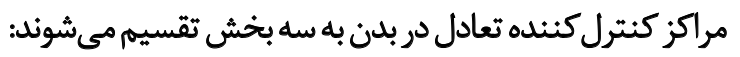

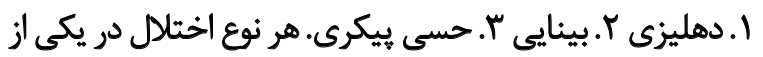
اين سيستمها و يا عواملى مثل كاهش قدي قدرت عضلانى و كاهش

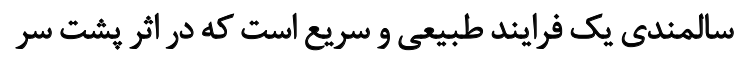

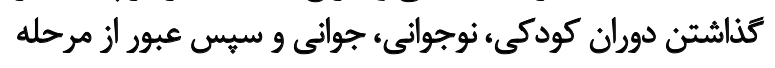

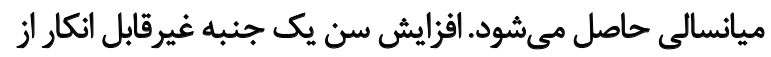

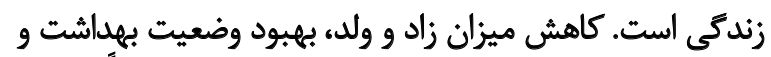

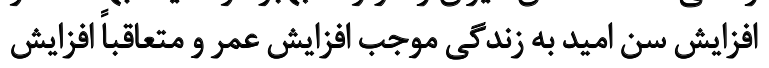

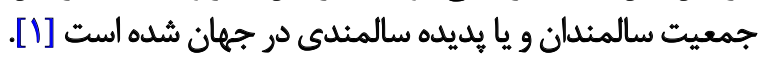
با شروع سالمندى تغييراتى در سيستمهاى فيزيولوزيك مرتبط

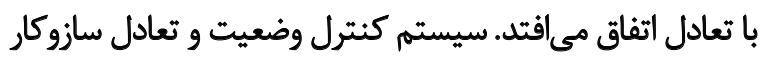

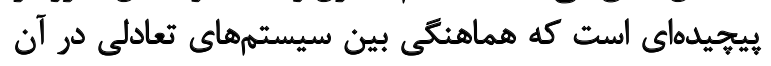
نقش بسزايى دارند [r].

ازجمله مشكلات شايع در سالمندان كه در اثر افزايش سن يا باري

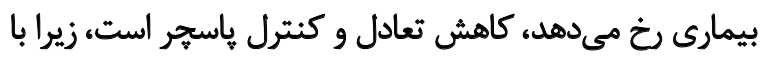
افزايش سن، تغييرات و يا نوسانات بدنى افزايش وني يافته و درنتيجه

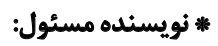

سيد مجتبى طباطباييى اصل

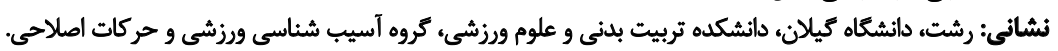

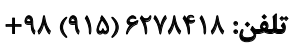
يست الكترونيكي: syd.mojtaba75@gmail.com 
اطلاعات اشتباه درباره موقعيت مفاصل و وضعيت فضايى بدن به به بادئ

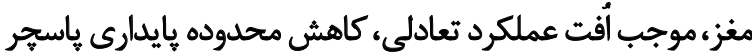

$$
\text { و أفت قابليت اصلاح در هاسجر شود }
$$

تأثيرات مختلف درمانى، ازجمله تمرينات كاوتورن كوكسى به به باتئ

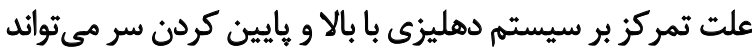

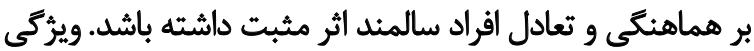

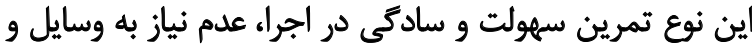

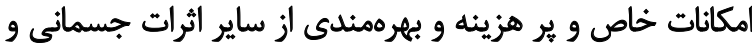

$$
\text { روانى فعاليتهاي بدنى است. }
$$

با توجه به توضيحات و مطالعات انجامشده در اين موضوع،

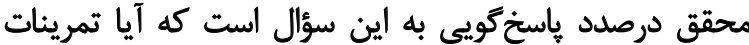

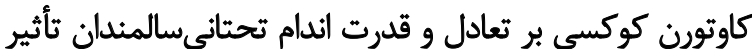

$$
\text { روششناسى }
$$

اين مطالعه از نوع نيمهتجربى و كاربردى بود كه در تابستان

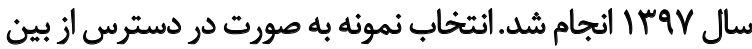

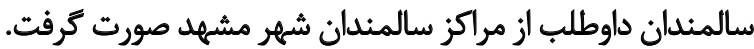

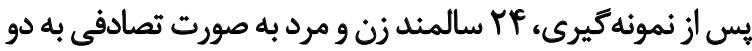
كروه دوازده نفرى تقسيم شدند.

توضيحات درخصوص نحوه اجراى تمرينات توسط محقق انجام

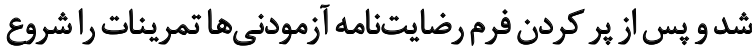

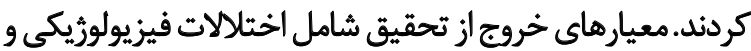

ياتولورى مانئد ياركينسون، ام اس، ناشنوايى، آلزايمر بود.

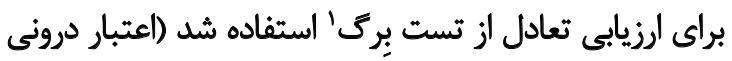

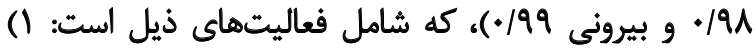

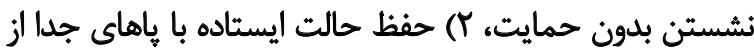

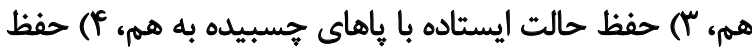

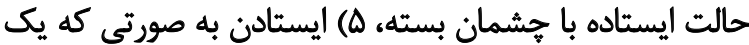

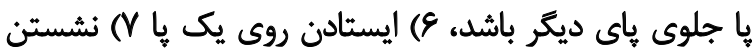

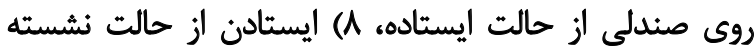

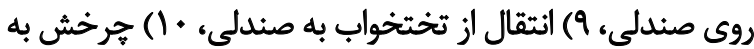

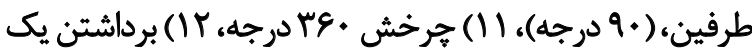

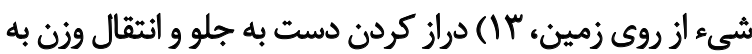

جلو و (If) انتقال وزن روى ياها به طور متناوب [IT)]

همجينين براي ارزيابى قدرت اندام تحتانى از تست زمان

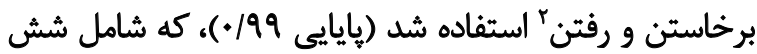

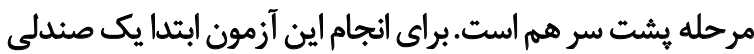
بدون دسته به فاصله سه مترى از يك مانع (يايان مسير) قرار داده إنائ

1. Berg Balance Scale

2. Tug Test
دامنه حركتى مي توانند اثر منفى روى تعادل فرد داشته باشد،

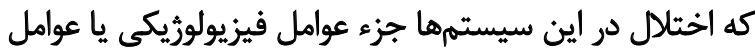

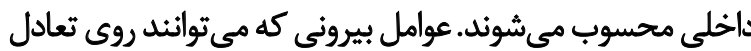

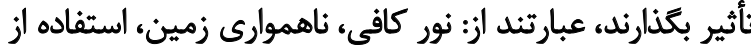

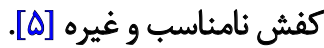
ميرمعزى و همكاران در تحقيقى به بررسى اثر تمرينات هوازي ئي

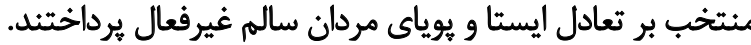

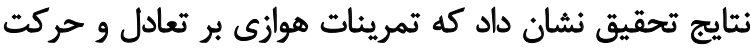

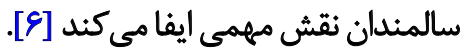

خواجوى و همكاران در تحقيقى به بررسى اثر تمرينات قدرتى و

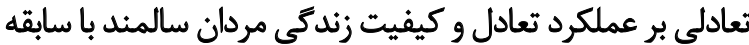

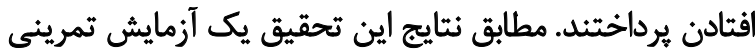

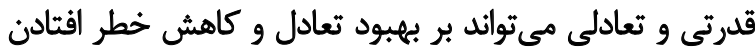

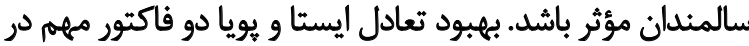

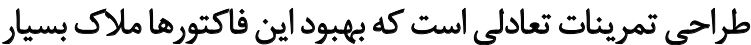

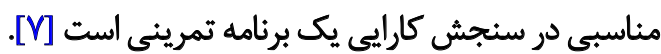
خورنا و همكاران در تحقيقى به بررسى اثر تمرينات كاوتورن

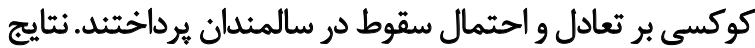

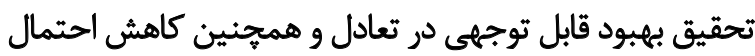
سقوط در سالمندان را نشان داد [ـ/].

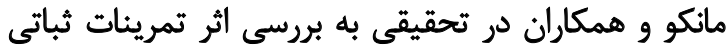

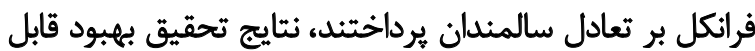

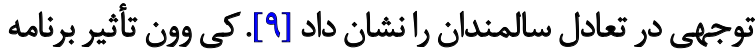

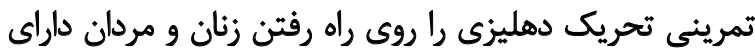

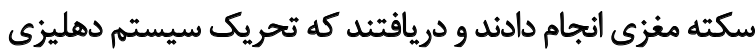

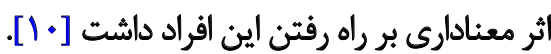
اقراد در بيشتر فعاليتهاى بدنى خود از يك جهتي يابي عمودى

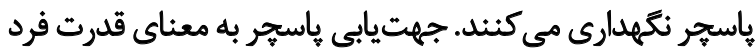

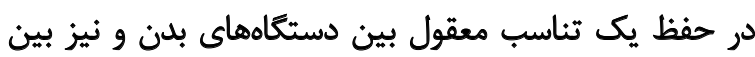

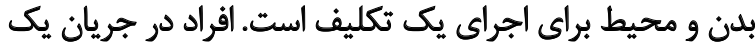

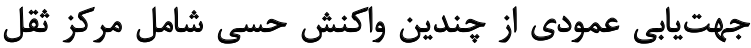

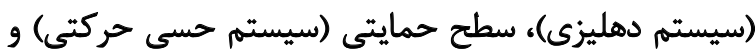
ارتباط بدن با اشيا، افراد و محيط اطراف (سيستم بينايي) استفاده

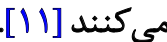

مطالعات نشان داده كه تغييرات سنى در زمينه

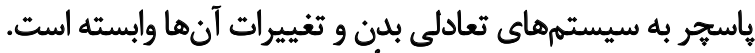

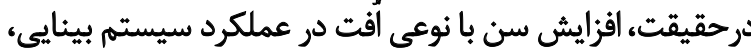

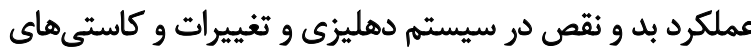
افزاينده در سيستم حس عمقى افر داد همراه است. اين امر مىتواند به درستي اطلاعات ارسالى أز اين سيستمهابه

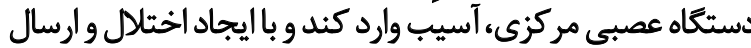


جدول ا. يروتكل تمرينى

\begin{tabular}{|c|c|c|c|c|c|c|c|}
\hline \multicolumn{2}{|c|}{ دو هفته جهارم } & \multicolumn{2}{|c|}{ دو هفته سوم } & \multicolumn{2}{|c|}{ دو هفته دوم } & \multicolumn{2}{|c|}{ دو هفته اول } \\
\hline זו تكراو & rا سِت & + ا تكرار & "إست & + أكرار & r بِت & 1 تكرار & r بِت \\
\hline
\end{tabular}

حركات جشم و سر، در حالت نشسته (ابتدا كند و بعد سريعتر):

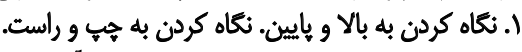

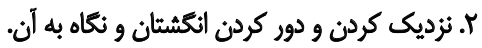

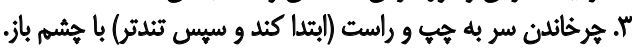

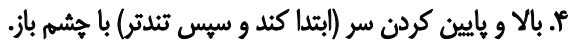

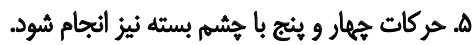

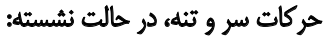

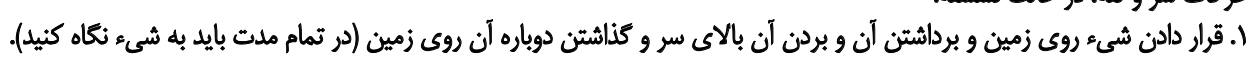

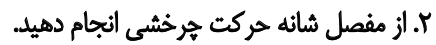

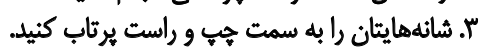
تمريثات ايستاده:

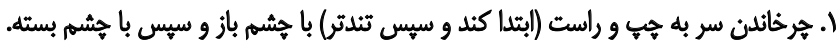

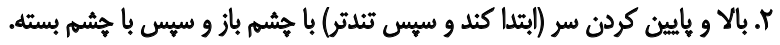

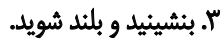

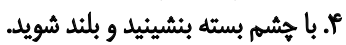

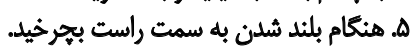

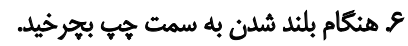

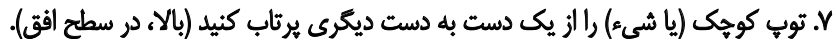

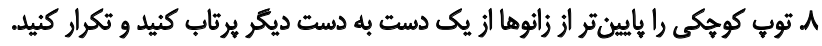

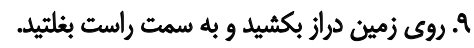

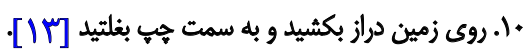

مجله بيومكانيك وزنش

نتئن

نتايج آزمون نرماليتى شاييرو ويلك، نرمال بودن دادهها را

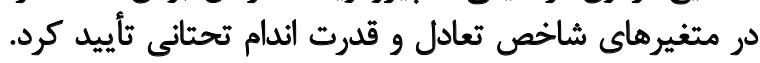

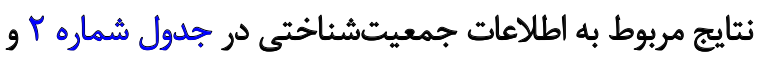

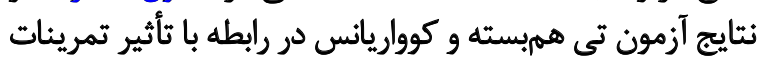
كاوتورن كوكسى بر تعادل و قدرت اندام تحتانى سالمئدان در

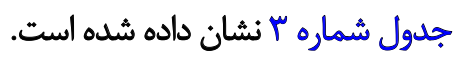

همان كونه كه در جدول شماره r مشاهده مي شود، در مرحله

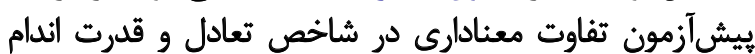

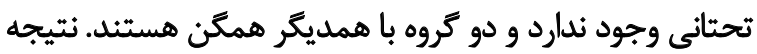

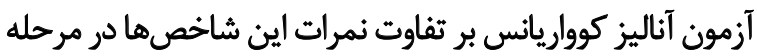
يسآزمون نشان داد كه بين دو كروه كنترل و آزمايش دئ در شاخد

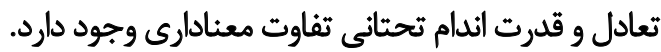

همجنين نتايج حاصل از آزمون تى وابسته نشان داد كه كروه

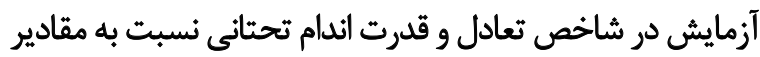

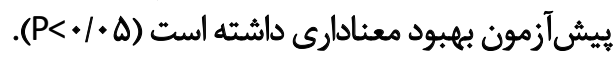

شده، فرد در حالت نشسته با شنيدن فرمان رو حركت كرده و

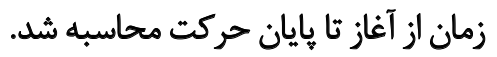

آزمودني ها بايد در سريعترين حالت ممكن و بلدون دويلن اين

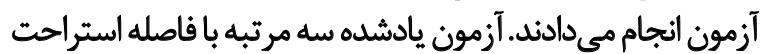

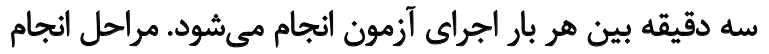

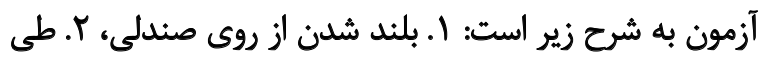

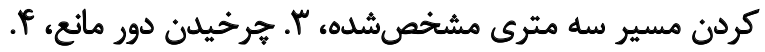

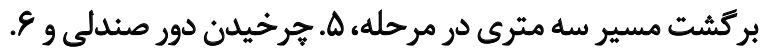

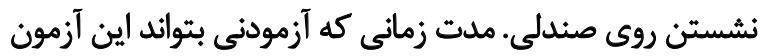

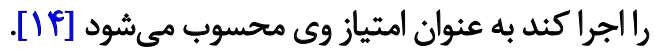

يس از اندازهيرىهاى يُشآزمون، افراد كروه آزمايش، سه

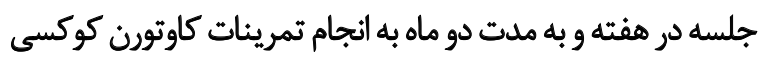

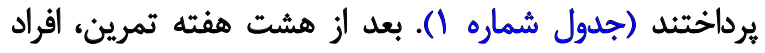

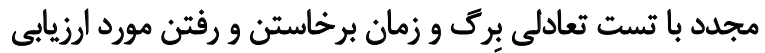

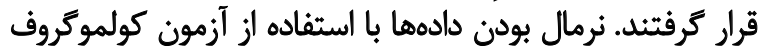

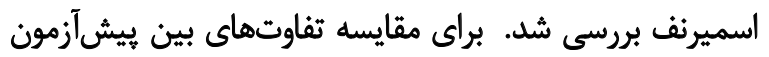

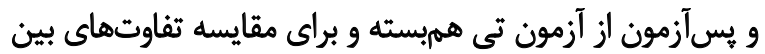

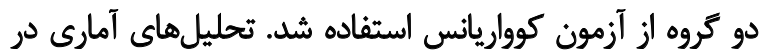

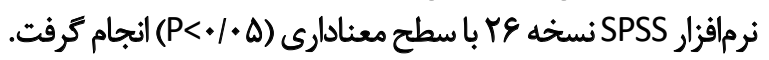


جدول r. ويرّكى هاي آنترويومتريكى آزموننىها

\begin{tabular}{|c|c|c|}
\hline \multicolumn{2}{|c|}{ ميانكين \ انحراف معيار } & \multirow{2}{*}{ مشخصات جمعيتشناختى } \\
\hline كروه كتترل (n-1Y) & كروه آزمايش (n=) (n) & \\
\hline EV/ITEVIRT & $\varepsilon \Delta / r \pm T / q \Delta$ & سن (تقويمى) \\
\hline $\mid F N \cdot P \pm \Delta / / Y$ & $19 q / 9 . \pm 8 / 97$ & قد (سائتىمتر) \\
\hline$Q V / M I \pm V / T F$ & ENI. $\pm 9 / A V$ & وزن (كيلوكرم) \\
\hline
\end{tabular}

مجله بيومكانيك وزنش

يافتهاى تحقيقات فرنادا و همكاران، كامرانى فراز و همكاران

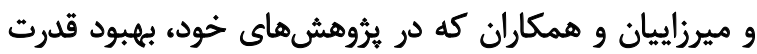

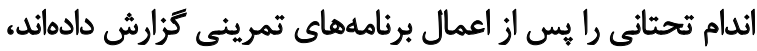

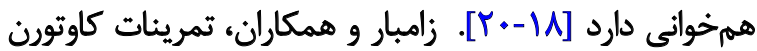

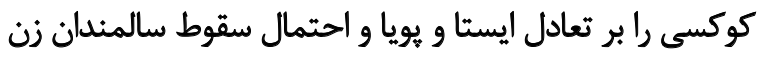

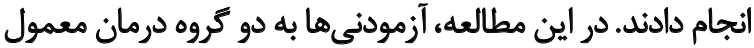
و كروه كاوتورن كوكسى و درمان تقسيم شدند.

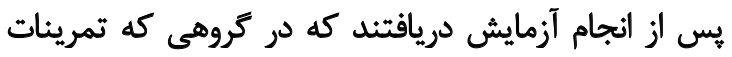

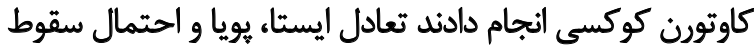

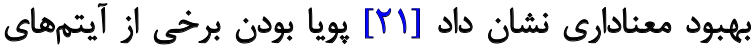

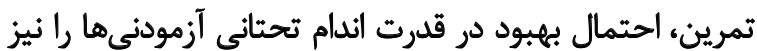
مي مهن.

از طرفى، به دليل اينكه آزمون برك به تنهايى نمى تواند بيانكر

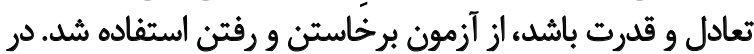

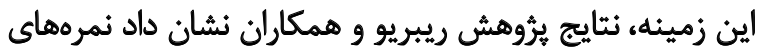

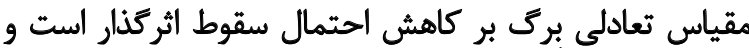

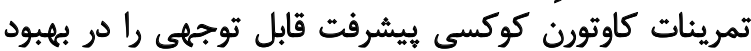

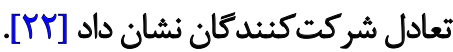
محمودى و همكاران، در بروهشى به اين نتيجه رسيدند كه

هدف از انجام يزوهش حاضر، بررسى تأثير تمرينات كاوتورن

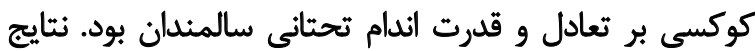

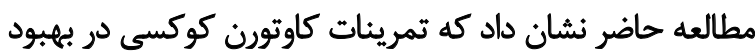

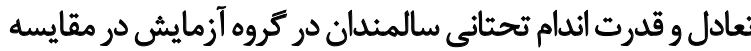

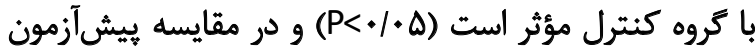

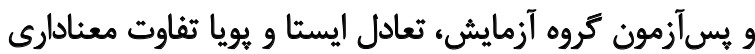

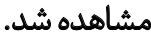

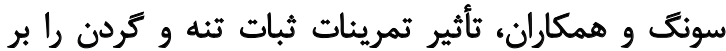

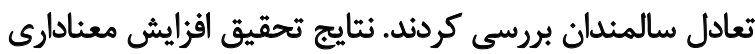

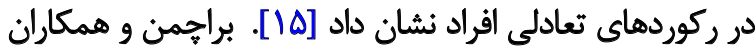

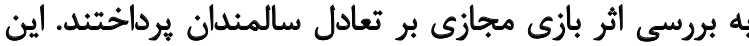

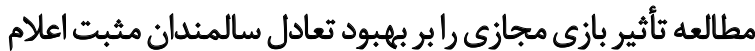

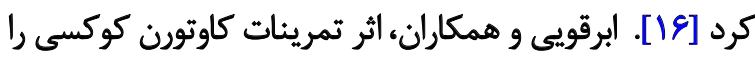

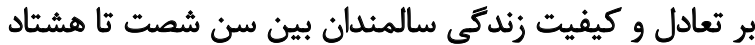

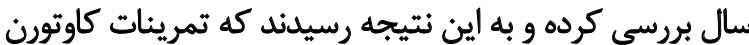

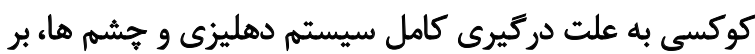

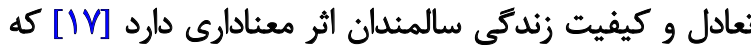

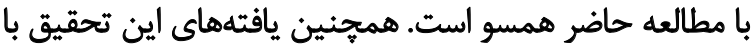

جدول ". تحليل نثايج درون كروهى و برون كروهى هر دو كروه تمرينى بر اساس آزمون تى وابسثه وكوواريانس

\begin{tabular}{|c|c|c|c|c|}
\hline \multicolumn{5}{|c|}{ ميانكيند|نحراف استاندارد } \\
\hline P** & يس آزمون & ييش آزمون & كروه & متغير \\
\hline $.1+1^{*}$ & $r+/ \Delta A \pm r / r \Delta$ & $r E / \Delta E \pm T / T r$ & آزمايش & \\
\hline . IPV & $r \Delta / \lambda \Psi \pm 1 / \varphi$. & relleEYAP & كتتل & \\
\hline - & $+1++*$ & $\cdot|| r \mid$ & - & "بين كروهى " \\
\hline $.1++r^{*}$ & $|\Delta / r+ \pm| / F \mid$ & $|e / f F \pm|(A)$ & أزمايش & \\
\hline$+/ T \Delta F$ & $|\Delta / \Delta \cdot \pm| / 8 \mid$ & ID/ATE $\pm / \Delta T$ & كنترل & قدرت أيدام تحتثائي \\
\hline - & $.1 \cdot \cdot v^{*}$ & $.1 \cdot W$ & - & 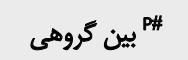 \\
\hline
\end{tabular}


حال كه در كشور ما ميانكين سن در حال افزايش است، نياز جامعه

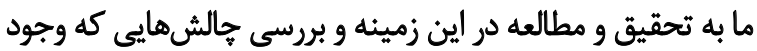

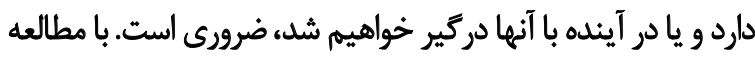

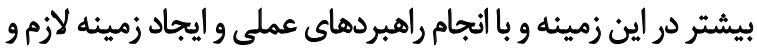

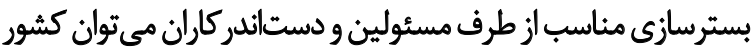

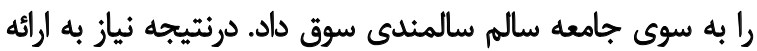

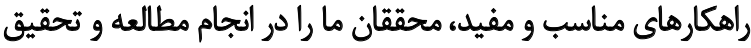

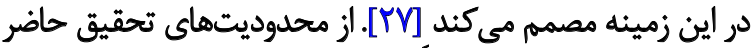

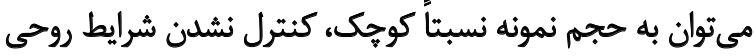

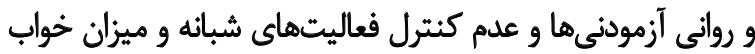
آزمودنى ها بر نتايج آزمون اشاره كرد.

\section{نتيجليرى نهايى}

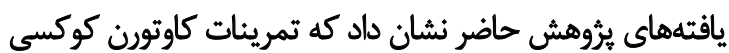

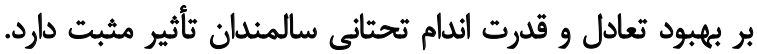

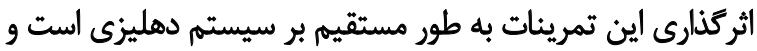

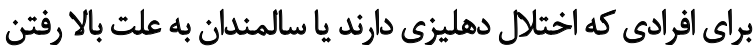

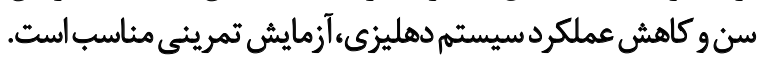

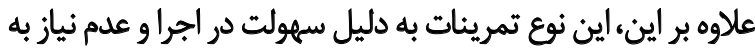

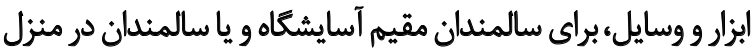

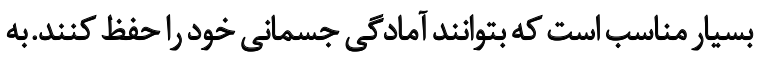

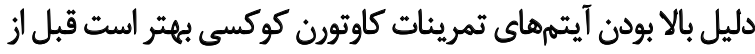

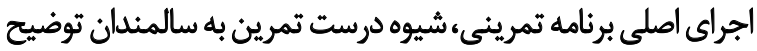

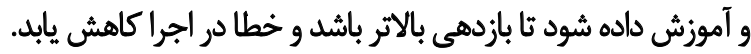

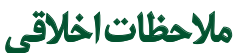

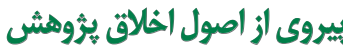

اصول اخلاقى تماماًدر اين مقاله رعايت شده است.شركت كنيندكان

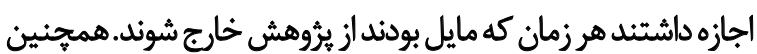

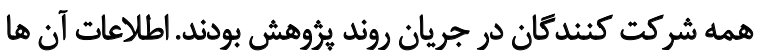

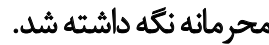

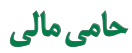

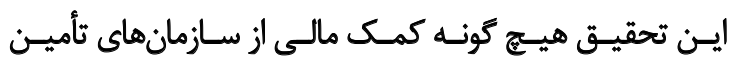

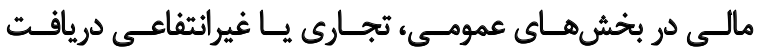

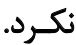

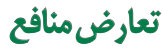

بنابر نظر نويسندكان، اين يُؤهش تعارض منافعى ندارد.
يك دوره تمرين ثبات مركزى بر بهبود تعادلٍ افراد سالمند تأثيركذار

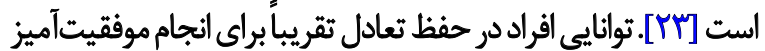

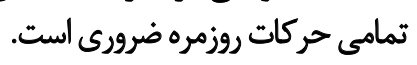

بر اساس تثئورى سيستمها، توانايى كنترل وضعيت بدن در فضان

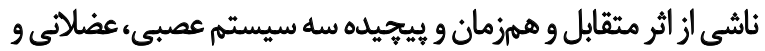

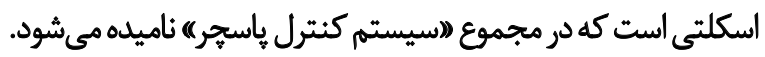

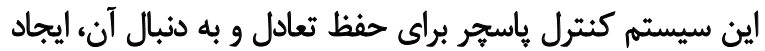

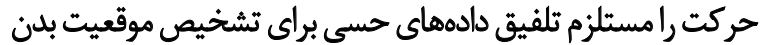

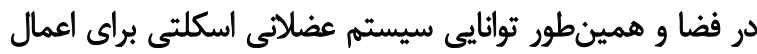

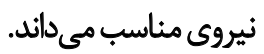
بر اساس اين تئورى، عوامل عضلاتى اسكلتى مؤثر در تنظيم

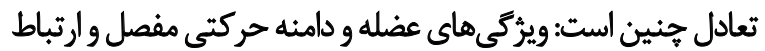

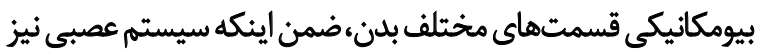
نقش بسيار مهمى در كنترل ياستير ايفا ميى كند. بئد

با استناد به تئورى سيستمهاو همجنين تأثير ورزش روى هريكاز

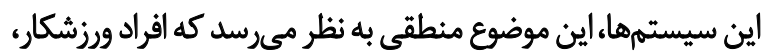

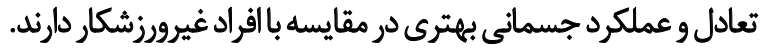

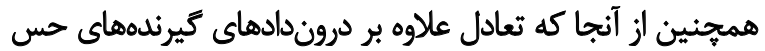

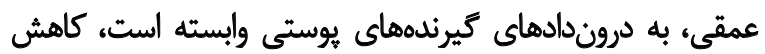

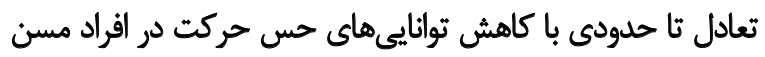

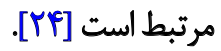

كاهش حداكثر قدرت عضلات نيز در افراد سالمند در نقصان كنترل

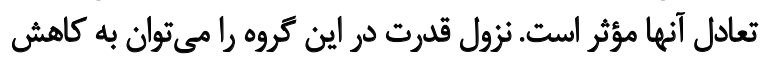

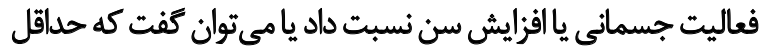

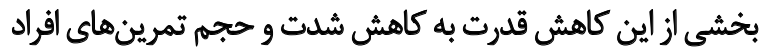

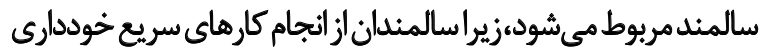

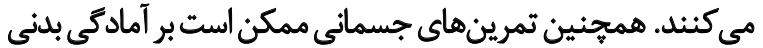

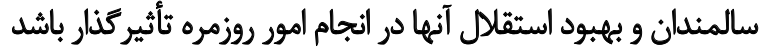

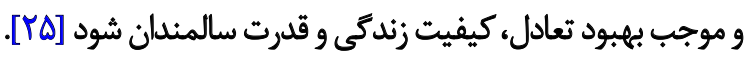

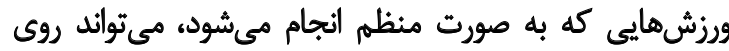

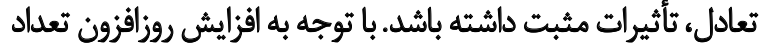

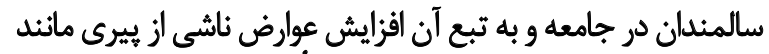

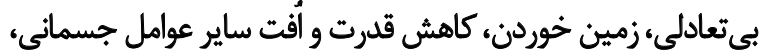

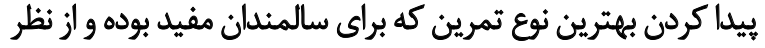

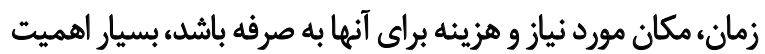

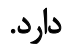

با توجه به تحقيقات انجامشده كه در بيشينه يثروهش بيان شد،

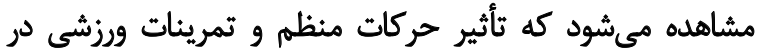

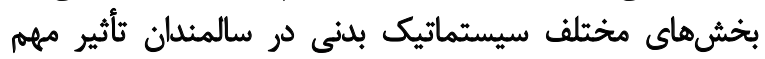
و جشمكير داشته و باعث بالا بردن سلامت سالمندان و درنيان دانتيجه سلامت خانواده و جامعه مي وشود [بعَ]. 


$$
\text { تشكرو قدردانى }
$$

يروزهشكران اين مطالعه وظيفه خود مي دانند از مسئولين

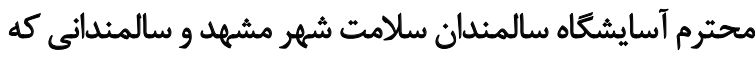

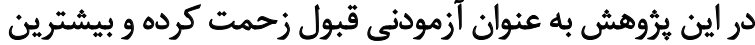

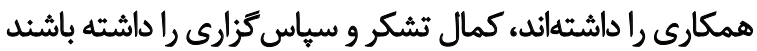




\section{Reference}

[1] Naeimikia M, Arab Ameri E, Ashayeri H, Hammayat Talab R, Azma $K$. The effect of external focus of attention instruction during walking training on old women's gait kinematic parameters. J Mot Learn Mov. 2011; 3(2):137-53. https://jmlm.ut.ac.ir/article_24642_6123ce6 6011e14f6c262f76c7b4783e8.pdf?lang=en

[2] Goudarz M, Foroughan M, Makarem A, Rashedi V. Relationship between social support and subjective well-being in older adults. Iran J Ageing. 2015; 10(3):110-9. https://salmandj.uswr.ac.ir/browse. php?a_id=953\&sid=1\&slc_lang=en

[3] Farsi A, Ashayeri $\mathrm{H}$, Mohammadzadeh $\mathrm{S}$. The effect of six weeks balance training program on kinematic of walking in women elderly people. Iran J Ageing. 2015; 9(4):278-87. http://salmandj.uswr.ac.ir/ browse.php?a_id=685\&sid=1\&slc_lang=en

[4] Beyranvand R. A survey on relationship between postural sway parameters and balance recovery strategies in older people. Daneshvar Med. 2016; 23(123):21-30. https://www.sid.ir/en/journal/ViewPaper. aspx?ID=505831

[5] Choi W, Joo Y, Lee S. Pilates exercise focused on ankle movement for improving gait ability in older women. J Women Aging. 2021 33(1):30-40. [DOI:10.1080/08952841.2019.1618129] [PMID]

[6] Mirmoezzi M, Amini M, Khaledan A, Khorshidi D. Effect of 8-week of selected aerobic exercise on static and dynamic balance in healthy elderly inactive men. Iran J Ageing. 2016; 11(1):202-9. [DOI:10.21859/ sija-1101202

[7] Khajavi D, Farokhi A, Jaberi Moghadam AK, Kazemnejad A. Effect of a strength and balance training program on maintaining balance and quality of life in older male adults with fear of fall. Iran J Ageing. 2016; 11(2):270-9. [DOI:10.21859/sija-1102270]

[8] Khurana N, Gaur DK, Linjhara S. Effect of Cawthorne and Cooksey ex ercises on balance in elderly and risk of fall. Indian J Gerontol. 2015 29(4):398-406. https://web.b.ebscohost.com/abstract?direct=true\&pr ofile=ehost\&scope=site\&authtype=crawler\&jrnl=09714189\&AN=1107 $20645 \& \mathrm{~h}=$

[9] Mańko G, Pieniążek M, Tim S, Jekiełek M. The effect of Frankel's stabilization exercises and stabilometric platform in the balance in elderly patients: A randomized clinical trial. Medicina. 2019; 55(9):583. [DOI:10.3390/medicina55090583] [PMID] [PMCID]

[10] Nam KW, Go JC, Yang YP. Effects of a vestibular stimulation training program on the gait of chronic stroke patients. Korean Soc Phys Med. 2019; 14(1):35-41. [DOI:10.13066/kspm.2019.14.1.35]

[11] Kiyani P, Farahpour N. Evaluation of performance of the vestibula proprioception and vision systems on postural control of old men. Iran J Ageing. 2015; 10(3):44-53. https://www.sid.ir/en/journal/ViewPaper.aspx?ID=580067

[12] Kashani VO, Zarifkar M, Alinaghipoor Z. Determining validity and reliability of the Persian version of Activities-specific Balance Confidence Scale for elderly. Koomesh. 2018; 20(4):705-12. http://koomeshjournal.semums.ac.ir/article-1-3728-en.html

[13] Feshki F, Banaei Far A, Kasbparast M. The effects of a 6-week selected balance and Cawthorne-Cooksey exercises on static balance and mobility in female patients with multiple sclerosis. Med J. 2020; 10(3):169-76. [DOI:10.32598/ptj.10.3.449.1]

[14] Asadi Samani Z, Rahnama N, Reisi J, Lenjan Nejadian S. Correlation between new activity-based balance index with accelerometer data and postural balance in elderly woman. Koomesh. 2020; 22(1):92-8. [DOI:10.29252/koomesh.22.1.92]
[15] Song GB, Park EC. Effects of neck and trunk stabilization exercise on balance in older adults. J Korean Physic Ther. 2016; 28(4):221-6. [DOI:10.18857/jkpt.2016.28.4.221]

[16] Brachman A, Marszałek W, Kamieniarz A, Michalska J, Pawłowsk $M, A k b a s ̧ A$, et al. The effects of exergaming training on balance in healthy elderly women-a pilot study. Int J Environ Res Public Health. 2021; 18(4):1412. [DOI:10.3390/ijerph18041412] [PMID] [PMCID]

[17] Abarghuei AF, Fadavi-Ghaffar M, Tousi S, Amini M, Salehi AR. Effect of cawthorne and cooksey exercises on balance and quality of life of 60 to 80 year-old individuals in Shiraz: A randomized clinical trial. Med J Islam Repub Iran. 2018; 23(4):32:74. [DOI:10.14196/ mjiri.32.74] [PMID] [PMCID]

[18] Fernanda de Mattos, Neiva Leite, Arthur Pitta, Paulo Cesar Barauce Bento. Effects of aquatic exercise on muscle strength and functional performance of individuals with osteoarthritis: A systematic review. Rev Bras Reumatol; 2016; 6(6):530-42. [DOI:10.1016/j. rbre.2016.09.003] [PMID]

[19] Kamranifaraz N, letafatkar A, Javdaneh N. The effect of a compound exercises in the water on muscle strength, flexibility, and quality of life of elderly women 60-70 years old in Tehran. Nursing J Vulnerable. 2017; 3(9):24-37. http://njv.bpums.ac.ir/article-1-751-en.html

[20] Mirzaeian E, Kazemzade Y, Sokhangooei Y. Effect of 8 weeks of water exercises on static and dynamic balance, walking, strength of the lower extremity of elderly women. Presented at: The first Nationa Conference of Sports Science Research New findings in the field of health, prevention, heroes and Tourism. 03 August 2017. Ahvaz; Shahid Chamran University of Ahvaz. https://www.en.symposia.ir/ CDLC01

[21] Zambare PD, Soni N, Sharma P. Effect of Cawthorne and Cooksey exercise program on balance and likelihood of fall in older women. Indian J Physiother Occup Ther. 2015; 9(3):55. [DOI:10.5958/09735674.2015.00095.7]

[22] Ribeiro AD, Pereira JS. Balance improvement and reduction of likelihood of falls in older women after Cawthorne and Cooksey exercises. Brazil J Otorhinolaryngol. 2015; 71(1):38-46. [DOI:10.1016/ S1808-8694(15)31283-0

[23] Mahmoudi S, Heyrani A. The effect of 8 weeks of core stability and core stability mindfulness exercises on the balance of older women in nursing centers of Kermanshah. J Motor Behav Sci. 2020; 3(3):247-56. http://www.jmbs.ir/article 118233.html?lang=en

[24] Jiakponnah NN, Unson C, Chukwurah QC. Correlates of strength and balance training exercises in older African American adults. Gerontologist. 2021; gnab016. [DOI:10.1093/geront/gnab016] [PMID]

[25] Gill TM, Pahor M, Guralnik JM, McDermott MM, King AC, Buford TW; LIFE Study Investigators, et al. Effect of structured physical activity on prevention of serious fall injuries in adults aged 70-89: Randomized clinical trial (LIFE Study). BMJ. 2016; 352:i245. [DOI:10.1136/ bmj.i245] [PMID] [PMCID]

[26] Farsi A, Abdoli B, Baraz P. The effect of balance, strength and combined training on balance in elderly women. Salmand. 2015 10(3):54-61. https://salmandj.uswr.ac.ir/article-1-726-en.html

[27] Behm DG, Muehlbauer T, Kibele A, Granacher U. Effects of strength training using unstable surfaces on strength, power and balance performance across the lifespan: A systematic review and metaanalysis. Sports Med. 2015; 45(12):1645-69. [DOI:10.1007/s40279015-0384-x] [PMID] [PMCID] 